\title{
Evolution of the Einstein Equations to Future Null Infinity
}

\author{
Oliver Rinne and Vincent Moncrief
}

\begin{abstract}
We describe recent progress with a formulation of the Einstein equations on constant mean curvature surfaces extending to future null infinity. Long-time stable numerical evolutions of an axisymmetric gravitationally perturbed Schwarzschild black hole have been obtained. Here we show how matter can be included in our formulation. We study late-time tails for the spherically symmetric EinsteinYang-Mills equations both for initial data that disperse and that collapse to a black hole.
\end{abstract}

\section{Introduction}

The standard approach to numerical simulations of asymptotically flat spacetimes is to adopt the Cauchy formulation of general relativity and truncate the spatial slices at a finite distance, where boundary conditions must be imposed. Apart from leading to a well-posed initial-boundary value problem, such boundary conditions should also be absorbing, i.e. they should be consistent with the solution on the unbounded domain. The problem is that the correct boundary conditions are not known at a finite distance. At best one may appeal to linearised theory. Bad choices of boundary conditions are known to destroy relevant features of the solution. A far more elegant approach is to include future null infinity in the numerical domain, which is the true physical boundary of spacetime. In order to do this, we follow

\footnotetext{
O. Rinne $(\square)$

Max Planck Institute for Gravitational Physics (Albert Einstein Institute), Am Mühlenberg 1, 14476 Potsdam, Germany

e-mail: oliver.rinne@ aei.mpg.de

V. Moncrief

Department of Mathematics and Department of Physics, Yale University,

New Haven, CT 06520, USA

e-mail: vincent.moncrief@yale.edu
} 
Penrose's approach and apply a conformal transformation to the spacetime metric, combined with a compactifying coordinate transformation. Rather than Friedrich's regular conformal field equations [1], we work directly with the Einstein equations in an ADM-like formulation on constant mean curvature (CMC) slices [2]. This formulation is reviewed in Sect. 2 and extended here to include matter sources. In Sect. 3 we review a first numerical implementation of this system, which achieved long-time stable evolution of a perturbed Schwarzschild black hole for the vacuum Einstein equations in axisymmetry. In Sect. 4 we include matter in the form of a Yang-Mills field, and we perform numerical simulations of the late-time decay of this field, restricted to spherical symmetry. Our evolutions include cases that form a black hole from regular initial data.

\section{General Formulation}

We decompose the spacetime metric ${ }^{(4)} g_{\mu v}$ in ADM form,

$$
{ }^{(4)} g=-N^{2} d t^{2}+g_{i j}\left(d x^{i}+X^{i} d t\right)\left(d x^{j}+X^{j} d t\right)
$$

where $g_{i j}$ is the induced metric on the $t=$ const slices, $N$ is the lapse function and $X$ the shift vector. The conformal spacetime metric ${ }^{(4)} \gamma_{\mu \nu}=\Omega^{2(4)} g_{\mu \nu}$ is decomposed in a similar way,

$$
{ }^{(4)} \gamma=-\tilde{N}^{2} d t^{2}+\gamma_{i j}\left(d x^{i}+X^{i} d t\right)\left(d x^{j}+X^{j} d t\right),
$$

where we identify $\gamma_{i j}=\Omega^{2} g_{i j}$ and $\tilde{N}=\Omega N$. The unit timelike normals of the physical and conformal spacetimes are related via $n^{\mu}=\Omega \tilde{n}^{\mu}$. The extrinsic curvature of the slices is defined as

$$
K_{i j}=-\frac{1}{2} \mathscr{L}_{n} g_{i j}
$$

where $\mathscr{L}$ denotes the Lie derivative. We require constant mean curvature,

$$
g^{i j} K_{i j} \equiv-K=\text { const }
$$

with $K>0$ so that the slices approach future null infinity $\mathscr{I}^{+}$. Our fundamental evolution variable is the traceless part of the ADM momentum

$$
\pi^{\operatorname{tr} i j}=-\mu_{g}\left(g^{i k} g^{j l}-\frac{1}{3} g^{i j} g^{k l}\right) K_{k l}
$$

where $\mu_{g}=\sqrt{\operatorname{det}\left(g_{i j}\right)}$.

Before continuing, we show how matter can be included in our formalism. We restrict ourselves to tracefree energy-momentum tensors, $g^{\mu \nu} T_{\mu \nu}=0$. Examples of matter models satisfying this condition include Maxwell and Yang-Mills fields 
and the conformally coupled scalar field. The tracefree condition insures that the matter evolution equations are conformally invariant (and hence are regular at $\mathscr{I}^{+}$), in particular,

$$
{ }^{(4)} \gamma^{\mu \nu(4)} \tilde{\nabla}_{\mu} \tilde{T}_{\nu \rho}=\Omega^{-4(4)} g^{\mu \nu(4)} \nabla_{\mu} T_{\nu \rho}=0,
$$

where we have introduced a conformally rescaled energy-momentum tensor $\tilde{T}_{\mu \nu} \equiv$ $\Omega^{-2} T_{\mu \nu}$. For this conformal energy-momentum tensor we introduce the usual projections

$$
\tilde{\rho} \equiv \tilde{n}^{\mu} \tilde{n}^{\nu} \tilde{T}_{\mu \nu}, \quad \tilde{J}^{i} \equiv-\gamma^{i \mu} \tilde{n}^{\nu} \tilde{T}_{\mu \nu}, \quad \tilde{S}_{i j} \equiv \gamma_{i}{ }^{\mu} \gamma_{j}{ }^{\nu} \tilde{T}_{\mu \nu}, \quad \tilde{S} \equiv \gamma^{i j} \tilde{S}_{i j} .
$$

We are now ready to write down the ADM equations. Let $\tilde{\nabla}$ denote the LeviCivita connection of $\gamma, \tilde{R}_{i j}$ its Ricci tensor and $\tilde{R}$ the Ricci scalar. The (not generally constant) mean curvature of the slices in the conformal spacetime is denoted by $\tilde{K}$. The evolution equations are

$$
\begin{aligned}
\mathscr{L}_{\tilde{n}} \gamma_{i j}= & 2 \mu_{\gamma}^{-1} \gamma_{i k} \gamma_{j l} \pi^{\operatorname{tr} k l}-\frac{2}{3} \gamma_{i j} \tilde{K} \\
\mathscr{L}_{\tilde{n}} \pi^{\operatorname{tr} i j}= & -2 \mu_{\gamma}^{-1} \gamma_{k l} \pi^{\operatorname{tr} i k} \pi^{\operatorname{tr} j l}-\frac{2}{3} \Omega^{-1} K \pi^{\operatorname{tr} i j} \\
& +\mu_{\gamma}\left[\tilde{N}^{-1} \tilde{\nabla}^{i} \tilde{\nabla}^{j} \tilde{N}-\tilde{R}^{i j}-2 \Omega^{-1} \tilde{\nabla}^{i} \tilde{\nabla}^{j} \Omega+\kappa \Omega^{2} \tilde{S}^{i j}\right]^{\operatorname{tr}} .
\end{aligned}
$$

The Hamiltonian and momentum constraints read

$$
\begin{aligned}
0= & -4 \Omega \tilde{\nabla}^{i} \tilde{\nabla}_{i} \Omega+6 \gamma^{i j} \Omega_{, i} \Omega, j-\Omega^{2} \tilde{R}-\frac{2}{3} K^{2} \\
& +\Omega^{2} \mu_{\gamma}^{-2} \gamma_{i k} \gamma_{j l} \pi^{\operatorname{tr} i j} \pi^{\operatorname{tr} k l}+2 \kappa \Omega^{4} \tilde{\rho}, \\
0= & \tilde{\nabla}_{j}\left(\Omega^{-2} \pi^{\operatorname{tr} i j}\right)+\kappa \mu_{\gamma} \tilde{J}^{i} .
\end{aligned}
$$

We also have an elliptic equation for the lapse arising from the constant mean curvature condition (4),

$$
\begin{aligned}
0= & -\Omega^{2} \tilde{\nabla}^{i} \tilde{\nabla}_{i} \tilde{N}+3 \Omega \gamma^{i j} \tilde{N}_{, i} \Omega_{, j}-\frac{3}{2} \tilde{N} \gamma^{i j} \Omega_{, i} \Omega_{, j}+\frac{1}{6} \tilde{N} K^{2} \\
& -\frac{1}{4} \tilde{N} \Omega^{2} \tilde{R}+\frac{5}{4} \tilde{N} \Omega^{2} \mu_{\gamma}^{-2} \gamma_{i k} \gamma_{j l} \pi^{\operatorname{tr} i j} \pi^{\operatorname{tr} k l}+\frac{1}{2} \kappa \tilde{N} \Omega^{4}(\tilde{S}+2 \tilde{\rho}) .
\end{aligned}
$$

In [2] we fixed the spatial coordinates by imposing a (spatial) harmonic gauge condition. However, other choices are possible; for example, in Sects. 3 and 4 we use coordinates adapted to the symmetry. There is also a residual conformal gauge freedom inherent in the decomposition $\gamma_{\mu \nu}=\Omega^{2} g_{\mu \nu}$. In [2] we fixed this by requiring the conformal scalar curvature $\tilde{R}$ to be constant. For the explicit forms of the conformal metrics used in Sects. 3 and 4 there is no remaining conformal gauge freedom.

The evolution equation (9) is formally singular at $\mathscr{I}^{+}$, where $\Omega=0$. However in [2] we showed how the offending terms can in fact be evaluated at $\mathscr{I}^{+}$in a 
regular way. This makes use of the fact that the constraints (10)-(11) and the CMC slicing condition (12) are also degenerate at $\mathscr{I}^{+}$. On a given spatial slice, we choose spherical polar coordinates such that the cut of the slice with $\mathscr{I}^{+}$corresponds to $r=r_{+}=$const. We expand the fields in finite Taylor series in $r$ about $r_{+}$and substitute them in the degenerate elliptic equations. Thus we obtain the first three radial derivatives of $\Omega$ and the zeroth and first radial derivative of $\pi^{\text {tr } r i}$ at $\mathscr{I}^{+}$. With this information we can evaluate the formally singular terms in the evolution equation (9) explicitly, provided that necessary conditions for smoothness of $\mathscr{I}^{+}$are satisfied. These include the condition that $\mathscr{I}^{+}$be shear-free and were obtained earlier in [3]. We show that these regularity conditions are preserved under the time evolution. While our analysis in [2] assumed vacuum, it is easy to see that it is unaffected by the addition of the matter sources, as will be shown in a forthcoming paper [4].

\section{Axisymmetric Vacuum Gravity}

The first numerical implementation [5] of the scheme presented in Sect. 2 assumed vacuum and axisymmetry. The spatial conformal metric is written in quasi-isotropic coordinates as

$$
\gamma=e^{2 \eta \sin \theta}\left(d r^{2}+r^{2} d \theta^{2}\right)+r^{2} \sin ^{2} \theta d \phi^{2},
$$

where $\eta$ is a function of $t, r$ and $\theta$ only, $\partial / \partial \phi$ being the Killing vector. Preservation of this form of the metric under the time evolution implies a first-order elliptic system for the shift vector similar to the Cauchy-Riemann equations.

The numerical implementation is based on fourth-order finite differences on a logically Cartesian grid in $r$ and $\theta$. The grid is allowed to be non-uniform in $r$ in order to better resolve the steep gradients occurring near the horizon of the black hole spacetimes we consider. We use black hole excision, i.e. the inner boundary is placed just inside the horizon. This boundary is spacelike, so evolution equations do not require any boundary conditions there. The outer boundary is placed at $\mathscr{I}^{+}$, where the regularised form of the evolution equations is used, as outlined at the end of the previous section. One-sided derivatives are used at both boundaries. The constraint equations, CMC slicing condition and spatial gauge condition are solved at each time step using a nonlinear multigrid solver. The evolution equations are integrated in time using the method of lines with a fourth-order Runge-Kutta method.

As a first test problem, we evolve Schwarzschild spacetime. We use the Schwarzschild metric in constant-mean-curvature coordinates derived in [6] with parameters $M=1, K=\frac{1}{2}$ and $C=2$. The Schwarzschild solution has a flat spatial conformal metric, $\eta=0$ in (13). We were able to evolve this solution for times as long as $10^{3} M$ (and potentially longer) without any signs of instability, with approximate fourth-order convergence as expected.

Next, we include a gravitational wave perturbation. For this we choose $\eta$ to be a Gaussian centred at $r=0.5$ with width $\sigma=0.05$ and amplitude $A=10^{-4}$, initially at rest. For comparison, the black hole horizon is at $r=0.0635$ and $\mathscr{I}^{+}$is at $r=1$. 
Fig. $1 \ell=2$ contribution to the Bondi news function for a gravitationally perturbed Schwarzschild black hole (mass $M=1$ ) as a function of time. Numerical results for two different resolutions are shown, $\left(N_{r}, N_{\theta}\right)=(64,8)$ (dashed line) and $(128,16)$ (solid line)

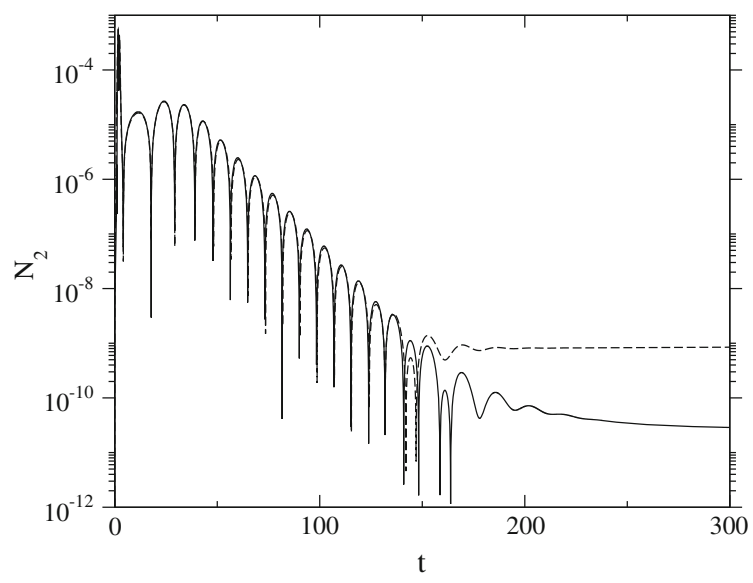

We stress that this perturbation is evolved using the full nonlinear Einstein equations rather than linearised theory.

We extract the gravitational radiation emitted by the system by evaluating the Bondi news function [7] at $\mathscr{I}^{+}$in Fig. 1. The quasi-normal mode ringing phase is clearly visible. The decay rate and frequency are consistent with the analytical result from linearised theory. At later times the numerical solution has not yet converged for the resolutions used here so we are currently unable to resolve the expected power-law tail.

\section{Spherically Symmetric Einstein-Yang-Mills}

In this section, we include matter in the form of a Yang-Mills field. This is conformally invariant; we choose to work in the conformal spacetime here. The energymomentum tensor is

$$
\tilde{T}_{\mu \nu}=\tilde{F}_{\mu \rho}^{(a)} \tilde{F}_{\nu}{ }^{\rho(a)}-\frac{1}{4}^{(4)} \gamma_{\mu \nu} \tilde{F}_{\rho \sigma}^{(a)} \tilde{F}^{\rho \sigma(a)},
$$

where the field-strength tensor $\tilde{F}_{\mu \nu}^{(a)}$ is given in terms of the connection $\tilde{A}_{\mu}^{(a)}$ by

$$
\tilde{F}_{\mu \nu}^{(a)}=\partial_{\mu} \tilde{A}_{\nu}^{(a)}-\partial_{\nu} \tilde{A}_{\mu}^{(a)}+f_{a b c} \tilde{A}_{\mu}^{(b)} \tilde{A}_{v}^{(c)} .
$$

Greek indices refer to the internal Yang-Mills gauge group, and the symbol $f_{a b c}$ is totally antisymmetric. Here we choose the gauge group to be $\mathrm{SU}(2)$, so Greek indices range over 1, 2, 3 and we may write $f_{a b c}=g[a b c]$, where $g$ is the YangMills coupling constant (taken to be $g=-2$ in the following) and [abc] is totally antisymmetric with [123] $=1$. 
Fig. 2 The Yang-Mills potential $F$ and electric field $D_{F}$ at $\mathscr{I}^{+}$(solid lines) and at the origin (dashed lines) in a subcritical evolution. The initial Bondi mass is 0.63

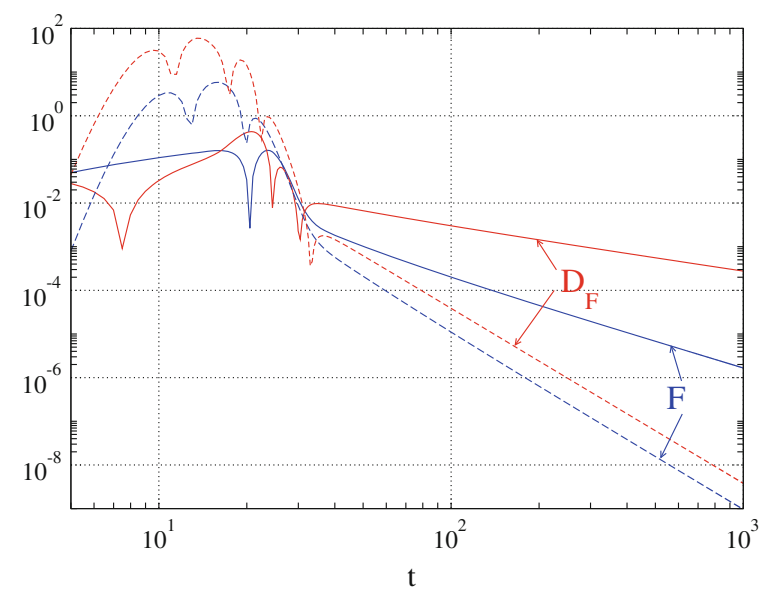

We now restrict ourselves to spherical symmetry. In this case we may always choose isotropic coordinates such that the spatial conformal metric is flat. This implies a first-order ordinary differential equation for the shift vector, which now has a radial component only. For the Yang-Mills connection we make the ansatz

$$
\tilde{A}_{i}^{(a)}=[a i j] x^{j} F(t, r), \quad \tilde{A}_{0}^{(a)}=0 .
$$

A more general spherically symmetric ansatz will be considered in [4]. Energymomentum conservation implies a nonlinear wave equation for $F$.

The numerical method used for this system is similar to the one described in Sect. 3. One difference is that in spherical symmetry, the traceless momentum $\pi^{\operatorname{tr} i j}$ only has one independent component, and we choose to solve the momentum constraint for it rather than its evolution equation. Hence the system is fully constrained and the only evolution equation used is the one for the Yang-Mills field. Also, our implementation allows for both regular and excised centres so that we may start from regular initial data until a black hole forms, which is then excised.

The Yang-Mills field $F$ is taken to be a Gaussian centred at $r=0.5$ with $\sigma=0.05$ (again, $\mathscr{I}^{+}$is at $r=1$ ) and variable amplitude. The time derivative of $F$ is chosen such that the pulse is approximately ingoing initially.

First we take the amplitude to be sufficiently small such that the field disperses. Figure 2 shows $F$ at the origin and at $\mathscr{I}^{+}$as a function of coordinate time. For the higher numerical resolution used in this $(1+1)$-dimensional simulation $\left(N_{r}=4000\right)$ the tail is now well resolved. At the origin (and in fact at any finite distance) the decay is approximately $F \sim t^{-4}$ whereas at $\mathscr{I}^{+}$, we find $F \sim t^{-2}$. This agrees with the results in [8], and the same decay exponents were found in the test field approximation [9]. 
Fig. 3 The Yang-Mills potential $F$ and electric field $D_{F}$ at $\mathscr{I}^{+}$(solid lines) and at the horizon (from when it forms, dashed lines) in a supercritical evolution. The initial Bondi mass is 3.0 and the final Bondi mass (which agrees with the final black hole mass) is 2.5

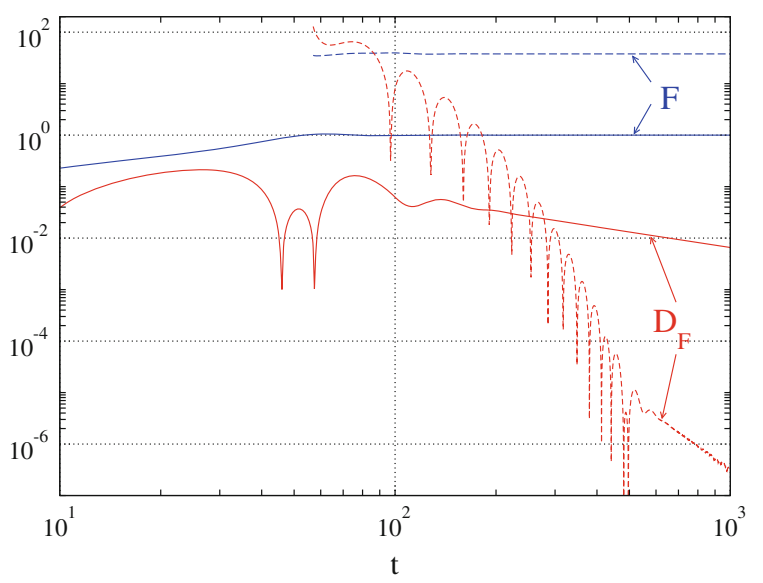

Let us also evaluate the electric field

$$
\tilde{D}^{i(a)}=\sqrt{-{ }^{(4)} \gamma} \tilde{F}^{0 i(a)} \equiv[a i j] x^{j} D_{F}(t, r),
$$

also shown in Fig. 2. While this decays at the same rate as $F$ at the origin, it decays more slowly at $\mathscr{I}^{+}, D_{F} \sim t^{-1}$. This may seem surprising at first but can be explained by looking at the evolution equation for $F$,

$$
F_{, t}=X^{r} F_{, r}+2 r^{-1} X^{r} F-\tilde{N} D_{F} .
$$

At $\mathscr{I}^{+}$the $r$-derivative of $F$ appearing on the right-hand side must decay more slowly than $F$ itself because $F$ decays faster away from $\mathscr{I}^{+}$. From (18) we infer that $D_{F}$ must also decay at the slower rate.

For sufficiently high amplitudes, the field collapses and a black hole forms (Fig. 3). Interestingly, the Yang-Mills potential $F$ does not decay to zero in this case but approaches $F=2 /\left(g r^{2}\right)$, which is another vacuum state (the field strength tensor vanishes). The electric field shows the same power-law decay as in the subcritical evolution.

Acknowledgments O.R. gratefully acknowledges support from the German Research Foundation through a Heisenberg Fellowship and research grant RI 2246/2. V.M. was supported by NSF grant PHY-0963869 to Yale University. 


\section{References}

1. Friedrich, H.: Cauchy problems for the conformal vacuum field equations in general relativity. Commun. Math. Phys. 91, 445 (1983). doi:10.1007/BF01206015

2. Moncrief, V., Rinne, O.: Regularity of the Einstein equations at future null infinity. Class. Quantum Grav. 26, 125010 (2009). doi:10.1088/0264-9381/26/12/125010

3. Andersson, L., Chruściel, P., Friedrich, H.: On the regularity of solutions to the Yamabe equation and the existence of smooth hyperboloidal initial data for Einstein's field equations. Commun. Math. Phys. 149, 587 (1992). doi:10.1007/BF02096944

4. Rinne, O., Moncrief, V.: Hyperboloidal Einstein-matter evolution and tails for scalar and YangMills fields, Class. Quantum Grav. 30, 095009 (2013). doi:10.1088/0264-9381/30/9/095009

5. Rinne, O.: An axisymmetric evolution code for the Einstein equations on hyperboloidal slices. Class. Quantum Grav. 27, 035014 (2010). doi:10.1088/0264-9381/27/3/035014

6. Brill, D., Cavallo, J., Isenberg, J.: K-surfaces in the Schwarzschild space-time and the construction of lattice cosmologies. J. Math. Phys. 21, 2789 (1980). doi:10.1063/1.524400

7. Bondi, H., van der Burg, M., Metzner, A.: Gravitational waves in general relativity. VII. Waves from axi-symmetric isolated systems. Proc. R. Soc. London, Ser. A 269, 21 (1962). doi:10.1098/ rspa.1962.0161

8. Pürrer, M., Aichelburg, P.: Tails for the Einstein-Yang-Mills system. Class. Quantum Grav. 26, 035004 (2009). doi:10.1088/0264-9381/26/3/035004

9. Zenginoğlu, A.: A hyperboloidal study of tail decay rates for scalar and Yang-Mills fields. Class. Quantum Grav. 25, 175013 (2008). doi:10.1088/0264-9381/25/17/175013 